\title{
Dipeptidase 1 (DPEP1) is a marker for the transition from low-grade to high-grade intraepithelial neoplasia and an adverse prognostic factor in colorectal cancer
}

\author{
P A Eisenach*,1, E Soeth ${ }^{2}$, C Röder ${ }^{3}$, G Klöppel ${ }^{4}$, J Tepel ${ }^{5}, \mathrm{H}$ Kalthoff $^{3}$ and B Sipos ${ }^{6}$ \\ ${ }^{1}$ Department of Molecular Medicine, Max Planck Institute of Biochemistry, Am Klopferspitz 18, 82152 Martinsried, Germany; \\ ${ }^{2}$ Clinical Research Centre Kiel GmbH, Schauenburgerstrasse 116, 24118 Kiel, Germany; ${ }^{3}$ Division of Molecular Oncology, Institute \\ of Experimental Cancer Research, University Hospital Schleswig Holstein, Arnold-Heller-Strasse 7, 24105 Kiel, Germany; \\ ${ }^{4}$ Department of Pathology, Technical University of Munich, Ismaninger Strasse 22, 81675 Munich, Germany; ${ }^{5}$ Department of \\ Surgery, Hospital Osnabrück, Am Finkenhügel 1, 49076 Osnabrück, Germany and 'Institute of Pathology and Neuropathology, \\ University Hospital Tübingen, Liebermeisterstrasse 8, 72076, Tübingen, Germany
}

Background: Colorectal cancer (CRC) is the second leading cause of cancer-related deaths worldwide. Improvements in the understanding of its molecular mechanism and the characterisation of CRC-specific biomarkers facilitating early detection are considered to increase overall survival.

Methods: A meta-analysis of microarray and Serial Analysis of Gene Expression (SAGE) has been performed to identify differentially regulated genes in CRC. Dipeptidase 1 (DPEP1/MDP/RDP) and Syntenin-2 (SDCBP2/SITAC18) were found to be differentially expressed in tumour tissue compared with normal mucosa. Expression of DPEP1 was assessed in a validation set of 87 normal mucosa samples, 20 hyperplastic polyps, 46 CR adenomas with low- and high-grade intraepithelial neoplasia (IEN) and 217 well-documented CRCs by immunohistochemistry and partially by immunoblotting and real-time PCR.

Results: Expression of DPEP1 was specifically increased in human CRC tissue samples compared with normal mucosa $(P<0.0001$, Mann-Whitney U-test), showing a striking upregulation in high-grade compared with low-grade IEN. Furthermore, high DPEP1 expression was found to strongly correlate with histological stage $(P<0.0001$, chi-square test) as well as localisation $(P<0.0001$, chi-square test) and has been recognised as an independent adverse prognostic factor, showing significant prognostic values with an ROC (receiver operating characteristic)-AUC of 0.9230.

Conclusion: Dipeptidase 1 has been identified as an excellent marker of high-grade IEN and CRC, and may thus be applied for screening of early neoplastic lesions and for prognostic stratification.

Colorectal cancer (CRC) is one of the most prevalent diseases and the second leading cause of cancer-related deaths in the western world (Siegel et al, 2012). The incidence of CRC is believed to result from a successive accumulation of genetic alterations spanning the development from benign adenomas to carcinomas
(Fearon and Vogelstein, 1990; Harrison and Benziger, 2011). Albeit the understanding of CRC's molecular signature is improving rapidly, resulting in more targeted treatments and a decline in cancer-related mortality in recent years (Jemal et al, 2010), clinically relevant molecular biomarkers for the specific and 
sensitive detection of CRC are still elusive (Duffy et al, 2007). Pathological staging relying on the Tumour-Node-Metastasis (TNM) system is currently the primary prognostic factor for patients with CRC (Wolpin and Mayer, 2008), which is, however, not accounting for the heterogeneity of individual tumours. Given that the survival of CRC patients is directly proportional to the tumour stage at the time of diagnosis (Siegel et al, 2011), there is an increasing demand for markers that are involved specifically in CRC progression, thus facilitating a more accurate prognostic stratification of the tumour to improve efficacy of multimodal therapy.

Membrane-bound dipeptidase 1 (DPEP1/MDP/RDP/Dehydropeptidase-I/Renal dipeptidase/EC3.4.13.19) is a zinc-dependent metalloproteinase that has been shown to process a plethora of peptides and antibiotics including thienamycin, penem and carbapenem derivates as well as to be involved in the glutathione and leukotriene metabolism (Kozak and Tate, 1982; Kropp et al, 1982; Nakagawa et al, 1992). Leukotrienes are known mediators of a variety of inflammatory diseases including asthma, arthritis and inflammatory bowel disease (Henderson, 1994a,b), making DPEP1 as their regulator a valuable target for therapeutic intervention. Although recent publications have acknowledged a link between DPEP1 mRNA expression levels and CRC (McIver et al, 2004; Okamoto et al, 2011; Toiyama et al, 2011), a comprehensive in vivo confirmation of a role of DPEP1 in patients with CRC remains pending.

In the present study, we provide evidence for the significant correlation of DPEP1 protein expression with CRC. Furthermore, we show DPEP1 (i) to be highly and specifically expressed in highgrade intraepithelial neoplasia (IEN) and CRCs and (ii) to correlate with adverse prognosis of CRC patients. This study describes DPEP1 for the first time as a potential tool for in vivo screening of early neoplastic lesions as well as supporting strategies towards personalised therapy.

\section{MATERIALS AND METHODS}

Human tissue samples and immunohistochemistry. Overall, 87 normal colonic mucosa, $20 \mathrm{CR}$ polyps, 31 adenomas with lowgrade IEN, 15 adenomas with high-grade IEN and 217 CRC samples were obtained at the University Hospital SchleswigHolstein (Kiel, Germany), frozen immediately after surgical resection and kept at $-80^{\circ} \mathrm{C}$ until further analysis. Tumour histopathology was classified according to the World Health Organization Classification of Tumour system. The present study was done with the approval of the local ethics committee (AZ 110/99). Immunohistochemistry was carried out on formalinfixed paraffin-embedded (FFPE) tissues according to routine methods. Anti-DPEP1 antibody, kindly provided by $\mathrm{N}$ Hooper (University of Leeds, UK), was applied in dilution of $1: 500$ without prior antigen retrieval. Detection was performed using biotin-based detection system by Vector Laboratories (Burlingame, CA, USA). Staining intensity was evaluated by an experienced pathologist (BS) according to a three-tier system as weak, moderate or strong, if at least $10 \%$ of cells showed DPEP1 staining. Hyperplastic polyps, adenomas showing various grades of IEN and 15 out of 22 T1 carcinomas were evaluated on conventional sections. Of the $148 \mathrm{CRC}$ cases represented on the tissue microarray (TMA), 147 specimens had sufficiently stained tissue to evaluate DPEP1 staining intensity. Among these tissue specimens, 117 cases were represented with three cores each while 26 cases had two cores on the TMA. Cases with only one core were omitted from further analysis $(n=4)$. In 53 cases, the DPEP1 score differed between cores taken from the same tissue specimen. Here, the modal score was taken. Where the modal score was not applicable, the highest score was used, which occurred in 13 cases. For Ki67/DPEP1 double staining, monoclonal anti-Ki67 antibody (MIb1; Dako Corp., Glostrup, Denmark) was used.

Real-time PCR. RNA isolation (peqGOLD RNAPure, PeqLab, Erlangen, Germany) and first-strand cDNA synthesis (Invitrogen, Karlsruhe, Germany) were performed according to the manufacturer's instructions. In all, $2 \mu \mathrm{g}$ of total RNA was reverse transcribed using random hexamer primers and the SuperScript II reverse transcriptase (Invitrogen). Sybr Green real-time PCR gene expression profiling was done using the real-time PCR iCycler (Bio-Rad, Munich, Germany). Reactions were performed in a 12.5$\mu \mathrm{l}$ final volume containing $6.5 \mathrm{ng} \mathrm{cDNA}, 500 \mathrm{~nm}$ of forward and reverse primer and SYBR Green Master Mix (Bio-Rad). To exclude potential genomic DNA contaminants and to ensure the specificity of the PCR, primers have been designed to be exon spanning (sequences in Supplementary Table 1). Additionally, a melting curve was performed following each PCR program and samples were subsequently loaded onto $2 \%$ Agarose gels to verify purity and size of amplicons. Fluorescent signal detection used ROX as the internal passive reference dye. Human GAPDH was used as a reference gene to normalise cellular RNA amounts. The relative expression of each sample was calculated using the $2^{-\Delta \Delta \mathrm{CT}}$ method.

Immunoblotting. Randomly selected tissue samples were homogenised and lysed in $0.1 \%(v / v)$ SDS, $1 \%(v / v)$ Nonidet P-40, $0.5 \%$ $(v / v)$ sodium desoxycholate, $1 \mathrm{~mm}$ EGTA and $1 \mathrm{~mm}$ sodium vanadate. Immunoblotting was performed as previously described (Eisenach et al, 2012). An anti-DPEP1 polyclonal antibody (kindly provided by NM Hooper; University of Leeds, UK) and the monoclonal anti- $\beta$-Actin antibody (Sigma-Aldrich, Munich, Germany) were used. Band intensities were quantified using the ImageJ image processing software (Schneider et al, 2012).

Statistical analysis. Statistical analysis was performed using the GraphPad Prism 5 Software (GraphPad Software, Inc., San Diego, CA, USA) and $R$ (R Core Team, 2012). Statistical significance was assessed by non-parametric Mann-Whitney $U$-test, unless indicated otherwise. All numerical values shown are means \pm s.e. Receiver operating characteristic (ROC) curves were constructed to distinguish CRC from normal tissue samples. To measure the significance of transcript expression of the Serial Analysis of Gene Expression (SAGE) libraries, the Fisher's exact test was used. To correct for multiple comparisons, the Benjamini-Hochberg method was used to calculate the false discovery rate $(Q)$. The survival probabilities were calculated by the Kaplan-Meier method and the differences between transcript expression groups were determined using the log-rank (LR) test. Prognostic factors were examined by univariate tests and the proportional hazard assumption was tested by using the $\mathrm{R}$ package 'survival' (Therneau, 2012).

\section{RESULTS}

Meta-analysis of SAGE and microarray data sets. To identify CRC prognostic markers, we sought to employ a meta-analysis of SAGE (SAGEmap, Cancer Genome Anatomy Project (CGAP)) data sets by the CGAP (Strausberg, 2001) and the human RZPD UniGene Set II microarray (Merck KgaA, Darmstadt, Germany). By using this combinational approach, we aimed to reduce the bias introduced by each method individually and to identify highly specific target genes associated with CRC development. Analysis of two CRC (N_Tu_102 and 98; 97071 transcript tags) and two normal colonic tissue (B_NC1 and 2; 98089 transcript tags) SAGE libraries with an expression factor cutoff of $F>2$ and a false discovery rate of $Q<0.1$ led to 350 differentially expressed 
transcript tags (Supplementary Table 2). These 350 targets were cross-referenced to the microarray data set, leading to a subset of 52 genes that were differentially regulated in CRC (Supplementary Table 3). A subsequent ingenuity pathway analysis (IPA) using UniGene identifiers revealed four statistically significant pathway networks that were differentially regulated in CRC (Supplementary Figure 1; Supplementary Table 3). Among these 52 potential target genes, 10 transcripts have been chosen based on (i) their significant differentially expression in CRC compared with controls, (ii) their biological function as mined by IPA and (iii) their intrinsic potential to be exploited as a drugable target (Table 1;

Table 1. Gene candidate validation in the discovery cohort $(n=10)$

\begin{tabular}{|l|c|c|c|}
\hline Gene target & $\begin{array}{c}\text { Expression } \\
\text { fold-change Tu/ } \\
\text { No (mean } \pm \text { s.e.) }\end{array}$ & $\begin{array}{c}95 \% \text { Cl of } \\
\text { mean }\end{array}$ & P-value \\
\hline SDCBP2 (SITAC18) & $0.07138( \pm 0.01554)$ & $0.03462-0.1081$ & 0.0142 \\
\hline RHOC (ARH9, ARHC) & $0.886( \pm 0.2214)$ & $0.3852-1.387$ & 0.3586 \\
\hline NMES1 (C15orf48) & $0.4106( \pm 0.1487)$ & $0.05883-0.7623$ & 0.0207 \\
\hline TM4SF1 & $4.495( \pm 1.378)$ & $1.377-7.612$ & 0.0137 \\
\hline TSPAN-1 & $0.5592( \pm 0.1516)$ & $0.2006-0.9177$ & 0.0391 \\
\hline IFI27 & $0.944( \pm 0.289)$ & $0.2789-1.610$ & 0.4028 \\
\hline OLFM4 (GW112) & $211.635( \pm 176.201)$ & $-187-610.2$ & 0.1934 \\
\hline LITAF (PIG7, SIMPLE) & $1.027( \pm 0.1826)$ & $0.6136-1.440$ & 0.8383 \\
\hline TBRG4 (CPR2, & $4.495( \pm 1.378)$ & $1.445-4.630$ & 0.0195 \\
FASTKD4) & $1023.9( \pm 803.345)$ & $-793.4-2841$ & 0.002 \\
\hline DPEP1 (MDP, RDP) & \multicolumn{3}{|l}{} \\
\hline Abbreviations: Cl=confidence interval; No=normal non-cancerous tissue; Tu $=$ tumour \\
tissue. Wilcoxon signed-rank test.
\end{tabular}

A

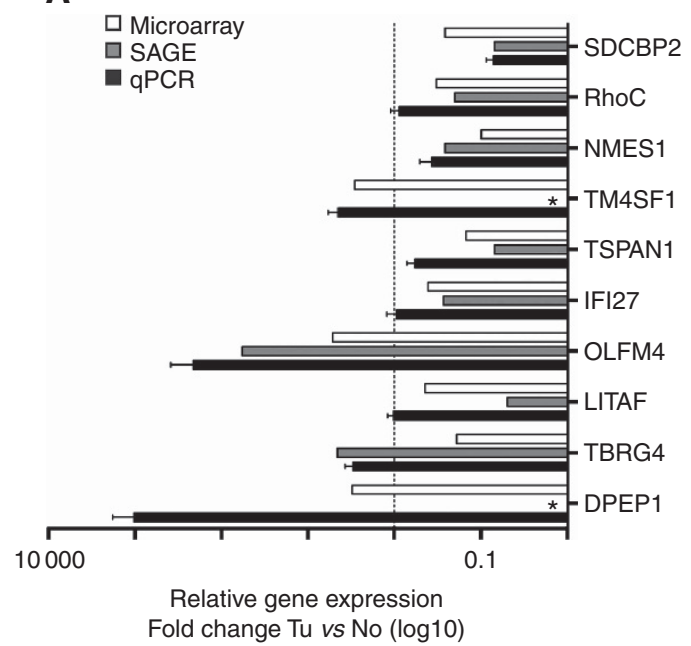

Supplementary Table 4). These candidates were validated by the virtual Northern tool in SAGEmap (Strausberg, 2001) and realtime PCR using 10 tissue pairs of CRC and adjacent normal mucosa (Discovery cohort; Figure 1A; Table 1). The SAGE and microarray results of all potential target genes were verified with DPEP1 and Syntenin-2 (SDCBP2) being the most significantly differentially expressed genes (Figure 1A). The association of DPEP1 and SDCBP2 levels in CRC was confirmed by univariate analysis of the discovery cohort and an extended cohort of $47 / 40$ cases (DPEP1/SDCBP2; validation cohort 1). The corresponding Hazard Ratios (HRs) with 95\% Confidence Intervals (CIs) and probabilities as determined by LR test are shown in Figure 1B.

DPEP1 and SDCBP2 mRNA is differentially expressed in tumour samples compared with corresponding normal tissue. Overall, 47/40 (DPEP1/SDCBP2) cases of the validation cohort 1 were used for further analysis. Tissue specimens were obtained at the University Hospital Schleswig-Holstein between 2001 and 2002. The overall survival data were completed in 2012. The demographic and clinicopathological characteristics are summarised in Tables 2 and 3. Quantitative real-time PCR analyses revealed a significant increase in DPEP1 (586.8 \pm 250.8 , mean \pm s.e., $P<0.0001$, Mann-Whitney $U$-test, normalised to GAPDH and normal tissue expression levels) and a reduction in SDCBP2 (0.2681 \pm 0.0759 , mean \pm s.e., $P<0.0001$, Mann-Whitney $U$-test, normalised to GAPDH and normal tissue expression levels) mRNA levels in tumour $v s$ corresponding normal tissue (Figure 2A). To evaluate the potential of DPEP1 and SDCBP2 as CRC-specific biomarkers, ROC curves were constructed. Both target genes could distinguish CRC from their corresponding controls with the following AUC: DPEP1, 0.9230 (95\% CI: 0.86560.9803; $P<0.0001 ; n=47$; Figure 2B) and SDCBP2, $0.7593(95 \%$ CI: $0.6491-0.8695 ; P<0.0001 ; n=40$; Figure $2 \mathrm{C}$ ). For analysing the association between overall patient survival and biomarker expression, Kaplan-Meier survival curves were generated on the

B

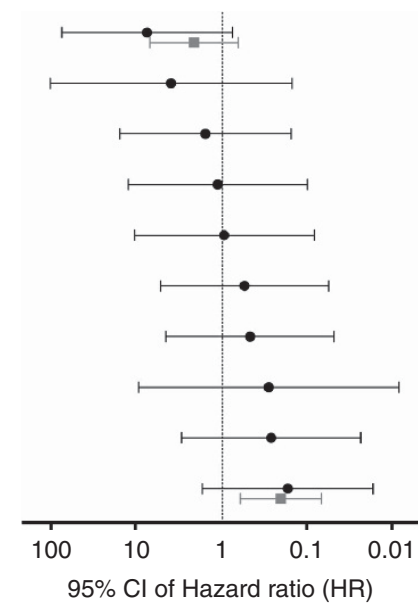

$\begin{array}{ll}\begin{array}{ll}\text { Discovery } \\ \text { cohort }\end{array} & \begin{array}{l}\text { Validation } \\ \text { cohort 1 }\end{array} \\ 0.0831 & 0.2099 \\ 0.4054 & \\ 0.1538 & \\ 0.9183 & \\ 0.9727 & \\ 0.6071 & \\ 0.5203 & \\ 0.4861 & \\ 0.2879 & \\ 0.1341 & 0.0048 \\ & \end{array}$

Figure 1. DPEP1 and SDCBP2 mRNA expression is differentially regulated in CRC compared with normal colonic mucosa. (A) The mRNA expression of downregulated and upregulated genes in CRC samples was compared between microarray (white bars), SAGE (grey bars) and realtime PCR (qPCR, black bars) analyses. For real-time expression profiling, the RNA of 10 pairs of tumour and control samples (Discovery cohort) was isolated, reverse transcribed and the expression levels of SDCBP2, RHOC, NMES1, TM4SF1, TSPAN1, IFI27, OLFM4, LITAF, TBRG4 and DPEP1 were determined. The mRNA expression levels of each candidate gene were normalised to GAPDH and to the expression level observed in noncancerous tissue. Data represent the mean mRNA expression plotted as the fold-change compared with control tissue (dotted line). ${ }^{*}$, no transcription tags were observed in non-cancerous control tissue in the SAGE analysis. Tu, tumour tissue; No, normal non-cancerous tissue. (B) The biomarker score was determined in the discovery cohort for all potential biomarker candidates (discovery cohort; black line) and in a set of 47 or 40 patients, respectively, for DPEP1 and SDCBP2 (validation cohort 1; grey line). Data represent the HR $\pm 95 \% \mathrm{Cl}$. The corresponding P-values of LR tests are indicated. 


\begin{tabular}{|c|c|c|c|c|}
\hline Variable & $\mathbf{N}$ & Mean ( \pm s.d.) & $\begin{array}{c}95 \% \mathrm{Cl} \text { of } \\
\text { mean }\end{array}$ & $P$-value \\
\hline Sex & & & & 0.789 \\
\hline Male & 26 & $548.8( \pm 1704)$ & $-139.6-1237$ & \\
\hline Female & 21 & $634.0( \pm 1779)$ & $-176.0-1444$ & \\
\hline Metastasis & & & & 0.7764 \\
\hline MO & 38 & $467.0( \pm 1432)$ & $-3.681-937.6$ & \\
\hline M1 & 9 & $1091( \pm 2677)$ & $-967.0-3149.0$ & \\
\hline $\begin{array}{l}\text { Lymph-node } \\
\text { metastasis }\end{array}$ & & & & 0.8901 \\
\hline NO & 36 & $488.1( \pm 1470)$ & $-9.118-985.4$ & \\
\hline $\mathrm{N} 1 / 2$ & 11 & $908.2( \pm 2428)$ & - 723.0-2539.0 & \\
\hline Localisation $^{\mathrm{a}}$ & & & & 0.0161 \\
\hline $\begin{array}{l}\text { Right colon/ } \\
\text { transversum }\end{array}$ & 17 & $95.16( \pm 161.9)$ & $11.92-178.4$ & \\
\hline Left colon/Sigma & 12 & $857.4( \pm 2317)$ & $-614.5-2329$ & \\
\hline Rectum & 18 & $870.9( \pm 2031)$ & $-139.1-1881$ & \\
\hline Age & & & & 0.932 \\
\hline$\leqslant 66$ & 22 & $613.4( \pm 1850)$ & $-206.9-1434$ & \\
\hline$>66$ & 25 & $563.5( \pm 1634)$ & $-111.1-1238$ & \\
\hline Grading $^{a}$ & & & & 0.2038 \\
\hline G1 & 4 & $112.9( \pm 67.31)$ & $5.778-220$ & \\
\hline G2 & 38 & $706.6( \pm 1897)$ & 83.11-1330 & \\
\hline G3 & 5 & $52.36( \pm 88.25)$ & $-57.22-161.9$ & \\
\hline Tumour stage $^{a}$ & & & & 0.0141 \\
\hline $\mathrm{T} 1 / 2$ & 16 & $286.2( \pm 414)$ & $65.62-506.8$ & \\
\hline T3 & 26 & $185( \pm 313.9)$ & $53.19-311.8$ & \\
\hline T4 & 5 & $3639.0( \pm 4438)$ & $-1872-9149$ & \\
\hline
\end{tabular}

basis of relative DPEP1 and SDCBP2 expression levels (cutoff= median expression level; Figure 2D and E). The mean survival period was 55 months in patients with high DPEP1 expression. We found a statistical significant correlation between DPEP1 expression and patient survival with $P=0.00481$ (LR) and an HR of 0.2069 (95\% CI: 0.06922-0.6186). The multivariable cox regression model with DPEP1 expression (dichotomised in high and low expression levels), lymph-node metastasis and metastasis variables found to be significant in univariate LR tests - rejected DPEP1 mRNA expression level as an independent prognostic variable in patients with CRC in this cohort (Figure 2D), indicating that DPEP1 mRNA expression does not provide an additional means of predicting patient outcome in this cohort. The mean survival period of SDCBP2 high/normal expressing patients was 53 months. However, albeit reduction in SDCBP2 expression was significantly linked to CRC (Figure 2A and C), we were not able to show a statistical significant correlation between SDCBP2 expression and patient survival in the cohort used $(P=0.2099$, LR; $\mathrm{HR}=2.147$, 95\% CI: 0.6503-7.091).

Correlation of DPEP1 and SDCBP2 expression with clinicopathological characteristics. To gain a better understanding of potential pathophysiological mechanisms, we examined the link between the expression level of both markers with clinicopathological and demographic variables including tumour localisation, stage, grade, metastasis, lymph-node status, sex, and
Table 3. Correlation of SDCBP2 expression and clinicopathological characteristics in validation cohort 1 ( $n=40$; fresh frozen tissue)

\begin{tabular}{|c|c|c|c|c|}
\hline Variable & $\mathbf{N}$ & Mean ( \pm s.d.) & $\begin{array}{c}95 \% \mathrm{Cl} \text { of } \\
\text { mean }\end{array}$ & $\boldsymbol{P}$-value \\
\hline Sex & & & & 0.9783 \\
\hline Male & 22 & $0.2111( \pm 0.2553)$ & $0.09791-0.3243$ & \\
\hline Female & 18 & $0.3378( \pm 0.6624)$ & $0.008428-0.6672$ & \\
\hline Metastasis & & & & 0.6433 \\
\hline MO & 33 & $0.2841( \pm 0.5159)$ & $0.1012-0.4671$ & \\
\hline M1 & 7 & $0.1927( \pm 0.2646)$ & $-0.05202-0.4374$ & \\
\hline $\begin{array}{l}\text { Lymph-node } \\
\text { metastasis }\end{array}$ & & & & 0.3349 \\
\hline NO & 32 & $0.2755( \pm 0.5165)$ & $0.08928-0.4617$ & \\
\hline $\mathrm{N} 1 / 2$ & 8 & $0.2386( \pm 0.3180)$ & $-0.02721-0.5045$ & \\
\hline Localisation $^{\mathrm{a}}$ & & & & 0.8007 \\
\hline $\begin{array}{l}\text { Right colon/ } \\
\text { transversum }\end{array}$ & 16 & $0.3313( \pm 0.6953)$ & $-0.006314-0.7018$ & \\
\hline $\begin{array}{l}\text { Left colon/ } \\
\text { Sigma }\end{array}$ & 8 & $0.2459( \pm 0.3017)$ & $-0.02746-0.4981$ & \\
\hline Rectum & 16 & $0.2161( \pm 0.2564)$ & $0.07948-0.3528$ & \\
\hline Age & & & & 0.1668 \\
\hline$\leqslant 66$ & 17 & $0.1745( \pm 0.2461)$ & $0.04795-0.3010$ & \\
\hline$>66$ & 23 & $0.3373( \pm 0.5938)$ & $0.08055-0.5941$ & \\
\hline Grading $^{\text {a }}$ & & & & 0.0753 \\
\hline G1 & 4 & $0.2428( \pm 0.3100)$ & $-0.2504-0.7361$ & \\
\hline G2 & 31 & $0.3075( \pm 0.5288)$ & $0.1135-0.5015$ & \\
\hline G3 & 5 & $0.04411( \pm 0.04729)$ & $-0.01461-0.1028$ & \\
\hline Tumour stage $^{a}$ & & & & 0.4811 \\
\hline $\mathrm{T} 1 / 2$ & 14 & $0.2087( \pm 0.2059)$ & $0.08985-0.3276$ & \\
\hline Т3 & 22 & $0.2988( \pm 0.6130)$ & $0.02702-0.5706$ & \\
\hline $\mathrm{T} 4$ & 4 & $0.3073( \pm 0.3949)$ & $-0.3212-0.9357$ & \\
\hline \multicolumn{5}{|c|}{$\begin{array}{l}\text { Abbreviations: } \mathrm{Cl}=\text { confidence interval; s.e. = standard error. } \\
\text { Mann-Whitney U-test. } \\
\text { a }_{\text {Kruskal-Wallis test }}\end{array}$} \\
\hline
\end{tabular}

age (Tables 2 and 3; Figure 3; Supplementary Figure 2). By using univariate analyses, we were not able to detect a significant correlation between any of the parameters outlined before and SDCBP2 expression (Table 3; Figure 3C and D). However, DPEP1 expression was found to be significantly associated with tumour localisation ( $P=0.0161$, Kruskal-Wallis test; Figure 3A) and stage $(P=0.0141$, Kruskal-Wallis test; Figure 3B).

DPEP1 protein expression correlates with tumour stage and localisation. Given the previous focus on the mRNA level and the restricted number of tissue samples analysed, we confirmed our observations by immunoblotting and immunohistochemistry. By using protein lysates of paired CRC and normal colonic mucosa samples (validation cohort 1), which were immunoblotted with an anti-DPEP1 antibody, we were able to confirm the increase in DPEP1 expression in tumour samples (Figure 4A, lower band). We routinely observed a non-specific band at $\sim 70-75 \mathrm{kDa}$, which most likely represents a cross-reaction in total tissue lysates, as this band is not present in protein lysates of cell lines (data not shown). Quantification of 13 representative immunoblots revealed a significant increase in DPEP1 expression in tumour samples compared with adjacent normal tissue (Figure 4A; DPEP1 expression normalised to $\beta$-actin expression levels; $0.3348 \pm 0.1126$ vs $1.079 \pm 0.2265 ; n=13$; mean \pm s.e.; $P<0.0001$, Student's $t$-test).

As we detected increased DPEP1 mRNA expression in T3/T4 vs T1/2 tumours, we sought to stain FFPE tissues of 10 normal 
A

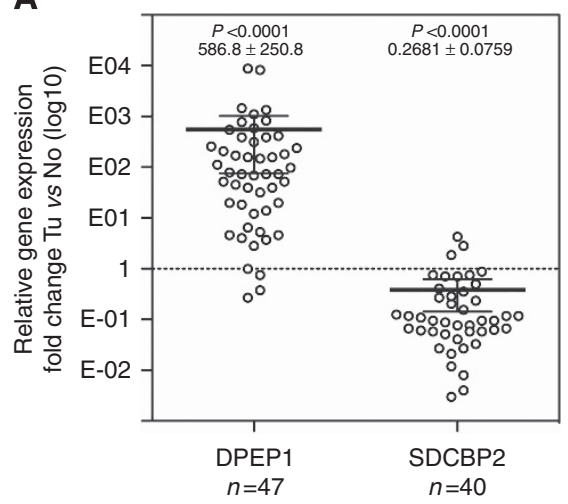

B

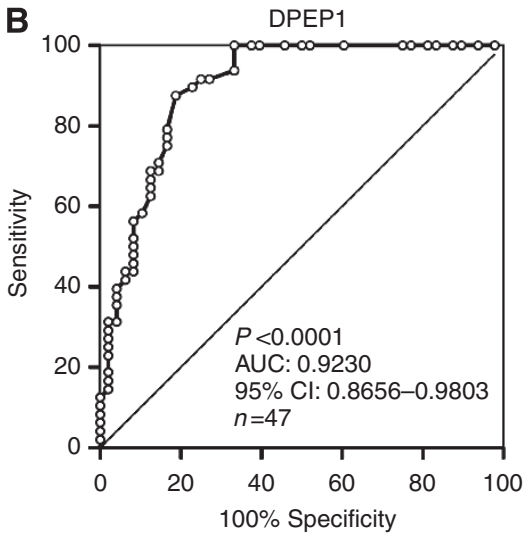

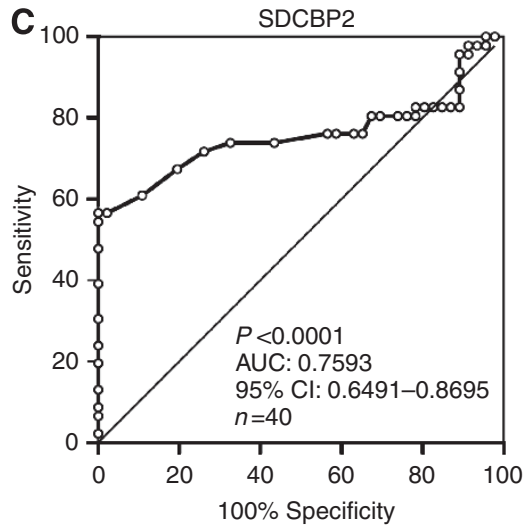

D

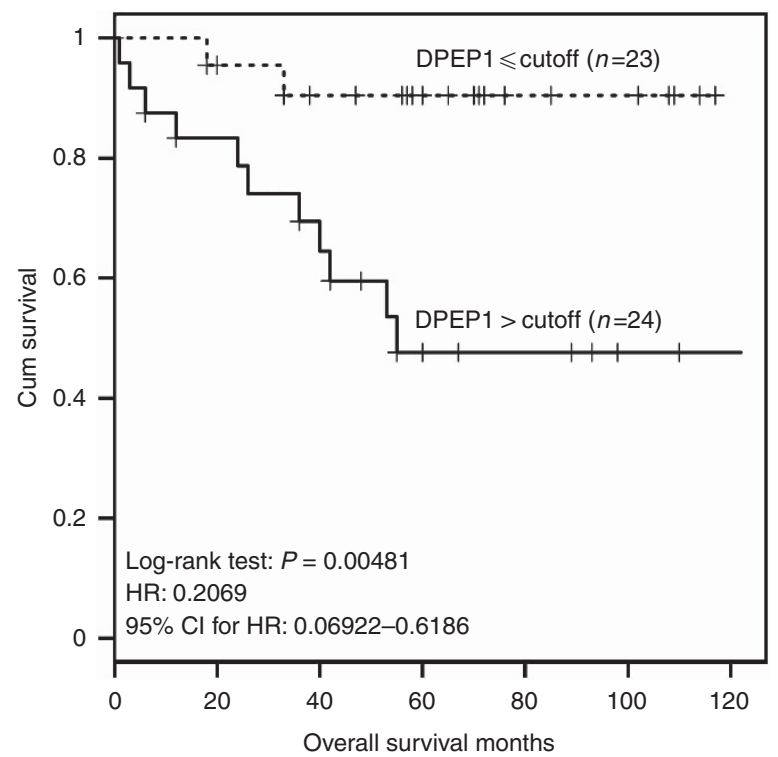

\begin{tabular}{|l|l|l|l|l|l|l|l|}
\hline Low & 23 & 20 & 17 & 12 & 6 & 5 & 0 \\
\hline High & 24 & 18 & 13 & 7 & 5 & 3 & 1 \\
\hline
\end{tabular}

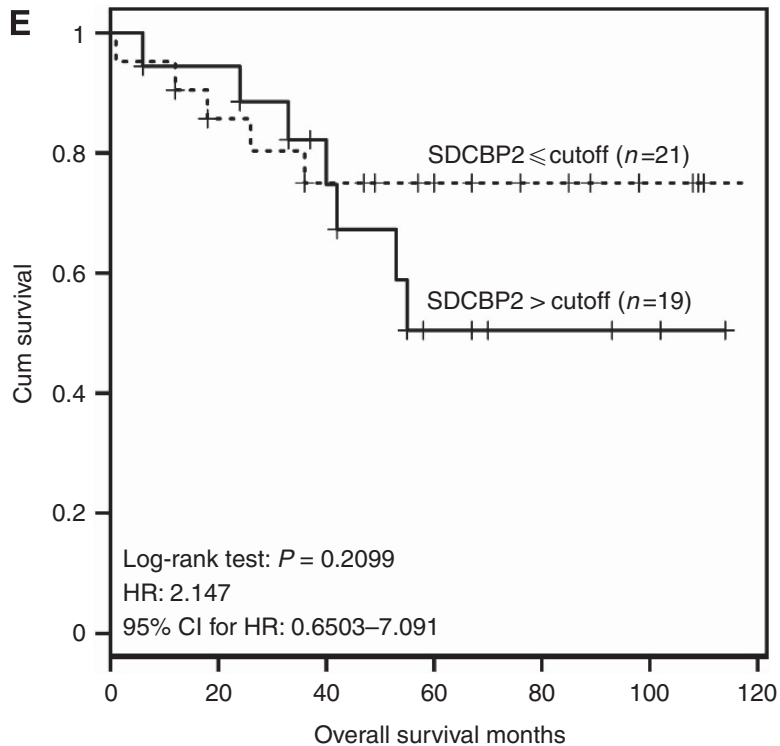

\begin{tabular}{|c|c|c|c|c|c|c|c|}
\hline Low & 21 & 16 & 14 & 10 & 7 & 4 & 0 \\
\hline High & 19 & 16 & 10 & 5 & 3 & 2 & 0 \\
\hline
\end{tabular}

\begin{tabular}{l|crc}
\hline Variable & Hazard ratio (HR) & $95 \%$ Cl for HR & $P$-value \\
\hline \hline DPEP1 expression (low vs high) & 0.2674 & $0.05243-1.3642$ & 0.113 \\
Lymph-node metastasis (NO vs N1/2) & 0.7358 & $0.19335-2.8004$ & 0.653 \\
Metastasis (M0 vs M1) & 0.1996 & $0.04876-0.8168$ & 0.025 \\
\hline
\end{tabular}

Figure 2. DPEP1 mRNA expression is a prognostic marker in CRC patients. (A) The mRNA of validation cohort 1 was isolated and the DPEP1 and SDCBP2 expression levels were analysed by real-time PCR. Relative expression levels of DPEP1 and SDCBP2 were normalised to GAPDH. Data represent the mean mRNA expression plotted as the fold-change compared with control tissue (dotted line; DPEP1: $n=47$ with $P<0.0001$; SDCBP2: $n=40$ with $P<0.0001$ ). The mean \pm s.e. is indicated. Tu, tumour tissue; No, normal non-cancerous tissue. (B and C) ROC curves of DPEP1 and SDCBP2 were created to differentiate CRC from normal controls (DPEP1: $n=47$; SDCBP2: $n=40$ ); the AUC and $95 \%$ Cl are indicated. (D and E) Kaplan-Meier survival curves for DPEP1 $(n=47)$ and SDCBP2 $(n=40)$ were generated. The numbers of patients at risk in each group at each time point for both markers are shown in the table below the respective plots. Patients with increased DPEP1 mRNA expression showed a significantly decreased overall survival rate $(P=0.004$, LR). The follow-up was 125 months, chosen cutoff value $=$ median expression level (D). Patients with lower SDCBP2 mRNA expression exhibited a non-significant increase in survival $(P=0.2099$, LR). The follow-up was 125 months, chosen cutoff $=$ median expression level $(\mathbf{E})$. Variables that were positive in univariate tests were used to generate a multivariable cox regression model. Only metastasis was an independent predictor of patient outcome. DPEP1 expression and lymph-node metastasis were rejected.

colonic mucosa, 20 hyperplastic polyps, 31 adenomas with lowgrade IEN, 15 adenomas with high-grade IEN, 22 CRCs as well as a TMA consisting of 30 normal mucosa and 148 CRC samples to refine the DPEP1 expression pattern during CRC progression. Among the 144 CRC samples present on the TMA with at least 2 cores, 2 cases were omitted due to either insufficient DPEP1 staining intensity or missing staging (Table 4). While tissue specimens obtained from normal mucosa (Figure 4Bi), hyperplastic polyps (Figure 4Bii) and tubular adenomas with low-grade IEN
(Figure 4Biii) exhibited low DPEP1 staining intensities, a highly significant upregulation of DPEP1 protein expression was observed during the transition to high-grade IEN (Figure 4Biv) and carcinomas (Figure $4 \mathrm{Bv}$ and vi). The increase in DPEP1 staining during the transition of low-grade towards high-grade IEN and carcinoma is shown in Figure $4 \mathrm{v}$. These observations were subsequently quantified revealing a highly significant correlation of DPEP1 expression and CRC progression $(P<0.0001$, chi-square test; Figure $4 \mathrm{C})$. Magnified micrographs depict the circumferential 

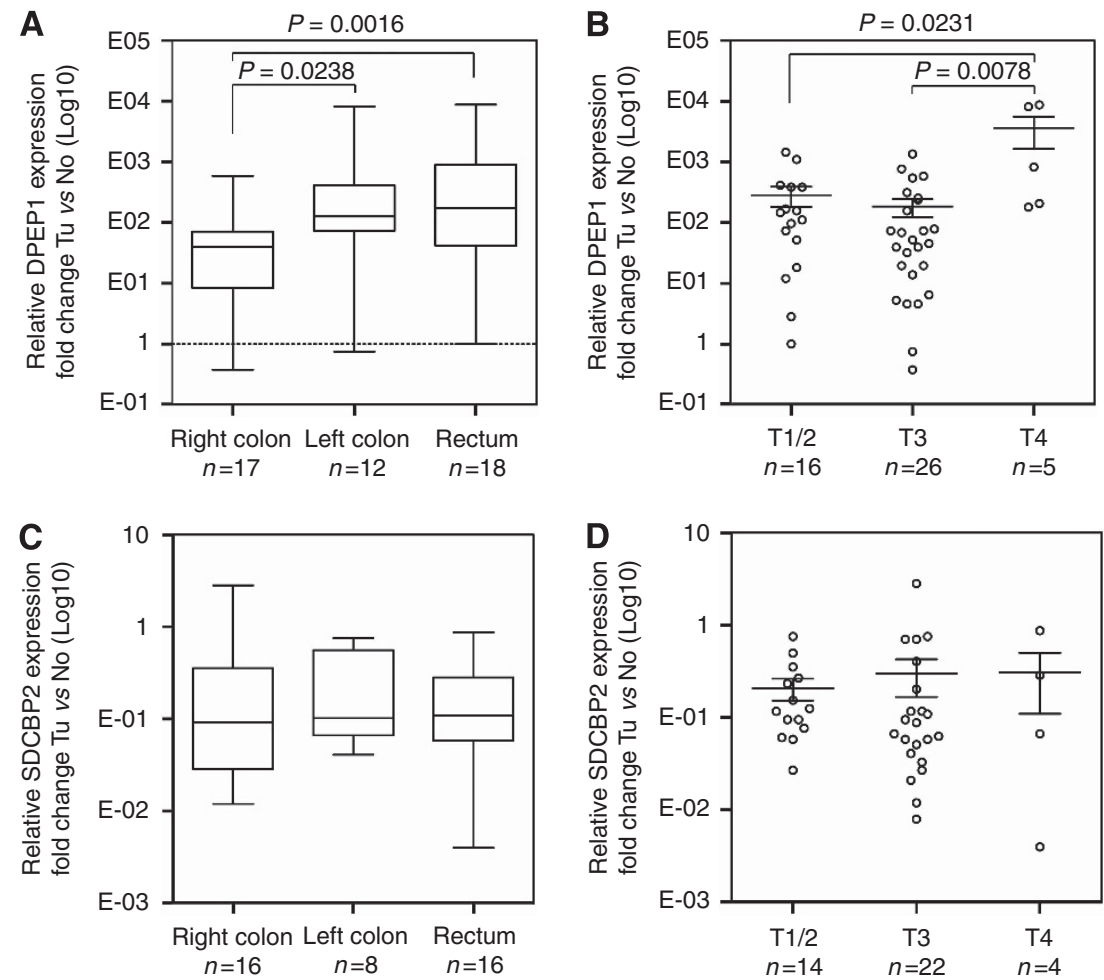

Figure 3. DPEP1 mRNA expression is associated with CRC localisation and stage. The mRNA of validation cohort 1 was isolated, reverse transcribed and the relative expression levels were analysed by real-time PCR. Relative expression levels of DPEP1 and SDCBP2 were normalised to GAPDH. Data represent the mean mRNA expression plotted as the fold-change compared with control tissue. (A and $\mathbf{C})$ Tumour localisation and ( $\mathbf{B}$ and $\mathbf{D}$ ) tumour stage. Tu, tumour tissue; No, normal non-cancerous tissue.

and apical staining pattern of DPEP1 (Figure 4Bvii-ix). Furthermore, we were able to confirm previous results obtained on the mRNA expression level by detecting an increased expression of DPEP1 protein within the left colon, the colon transversum and the rectum, compared with the right colon $(P<0.0001$; chi-square test, total analysed specimen: $n=111$; Figure $4 \mathrm{D})$.

DPEP1 expression is a prognostic marker for patient outcome. Taken together, 148 cases of CRCs operated at the University Hospital Kiel between 1994 and 2002 were analysed (validation cohort 2). The overall survival data for 126 cases were completed in 2012 with a mean follow-up time of 68.79 months. The demographic and clinicopathological characteristics of the total study cohort are summarised in Table 4. Kaplan-Meier plots were created of all DPEP1 score categories and the intensity was subsequently dichotomised into those with high-intensity staining (scores 2 and 3) and those with low-intensity staining (scores 0 and 1). Tumour stage and lymph-node status were found to be significant prognostic factors in univariable analysis. Cox regression analysis with tumour stage and lymph-node metastasis as input variables confirmed the significant role of DPEP1 as an independent prognostic marker of survival in the analysed patient cohort (Figure 5B).

\section{DISCUSSION}

The present paper represents the most comprehensive study on DPEP1's role in CRC on the protein level so far, providing sufficient statistical power to evaluate DPEP1 as an adverse prognostic factor. We identified elevated DPEP1 expression as a marker detecting high-grade IEN and CRCs with high specificity and sensitivity as well as correlated DPEP1 expression with adverse prognosis of CRC patients.
Although few publications have acknowledged a link between DPEP1 mRNA expression and carcinogenesis, its subsequent use as a tumour-specific prognostic marker has been controversial in recent years. Dipeptidase 1 has been identified as a potential tumour suppressor due to its decreased expression in Wilms' tumours (Austruy et al, 1993) and in invasive and in situ breast lobular carcinomas (Green et al, 2009). A recent publication demonstrated that DPEP1 expression inhibits tumour cell invasiveness in pancreatic ductal adenocarcinoma and negatively correlated with histological grade (Zhang et al, 2012). In contrast, DPEP1 has been found to be overexpressed in CRC cells (McIver et al, 2004; Yajima et al, 2007; Toiyama et al, 2011) and to act as a specific marker for disseminated tumour cells within blood samples in patients with CRC (McIver et al, 2004). These contradicting results indicate towards a potential tissue-specific role of DPEP1 during tumourigenesis as its expression is downregulated in Wilms' tumours, in invasive and in situ breast lobular carcinomas and pancreatic ductal adenocarcinomas (Austruy et al, 1993; Green et al, 2009; Zhang et al, 2012) vs an elevated DPEP1 expression in CRC (McIver et al, 2004; Toiyama et al, 2011; and this study). Considering further the different results obtained in the CRC-specific studies, there is a requirement to clarify the correlation between DPEP1 expression and clinicopathological parameters. In agreement with Toiyama et al (2011), we have detected enriched DPEP1 expression levels in tumour samples and in 5 out of 7 colon cancer cell lines (Supplementary Figure 3). However, in contrast to previous studies (McIver et al, 2004; Toiyama et al, 2011), we found DPEP1 to be specific for tumour localisation, to positively correlate with tumour stage and to be on the protein level - an independent prognostic marker of poor prognosis in patients with CRC. These differences observed might be primarily explained by the heterogeneity of the cohorts and the focus of previous studies on the mRNA level as opposed to our protein detection approach employed in the present work, as we 
have also found that DPEP1 is not an independent prognostic marker on the mRNA level.

Although having established DPEP1 as a prognostic marker for $\mathrm{CRC}$, its biochemical function during tumourigenesis remains elusive. Toiyama et al (2011) have postulated that expression of DPEP1, as a membrane-bound metalloproteinase, in CRC may be implicated in the degradation of the extracellular matrix during tumour progression, cell dissemination and metastasis. Although

\section{B}
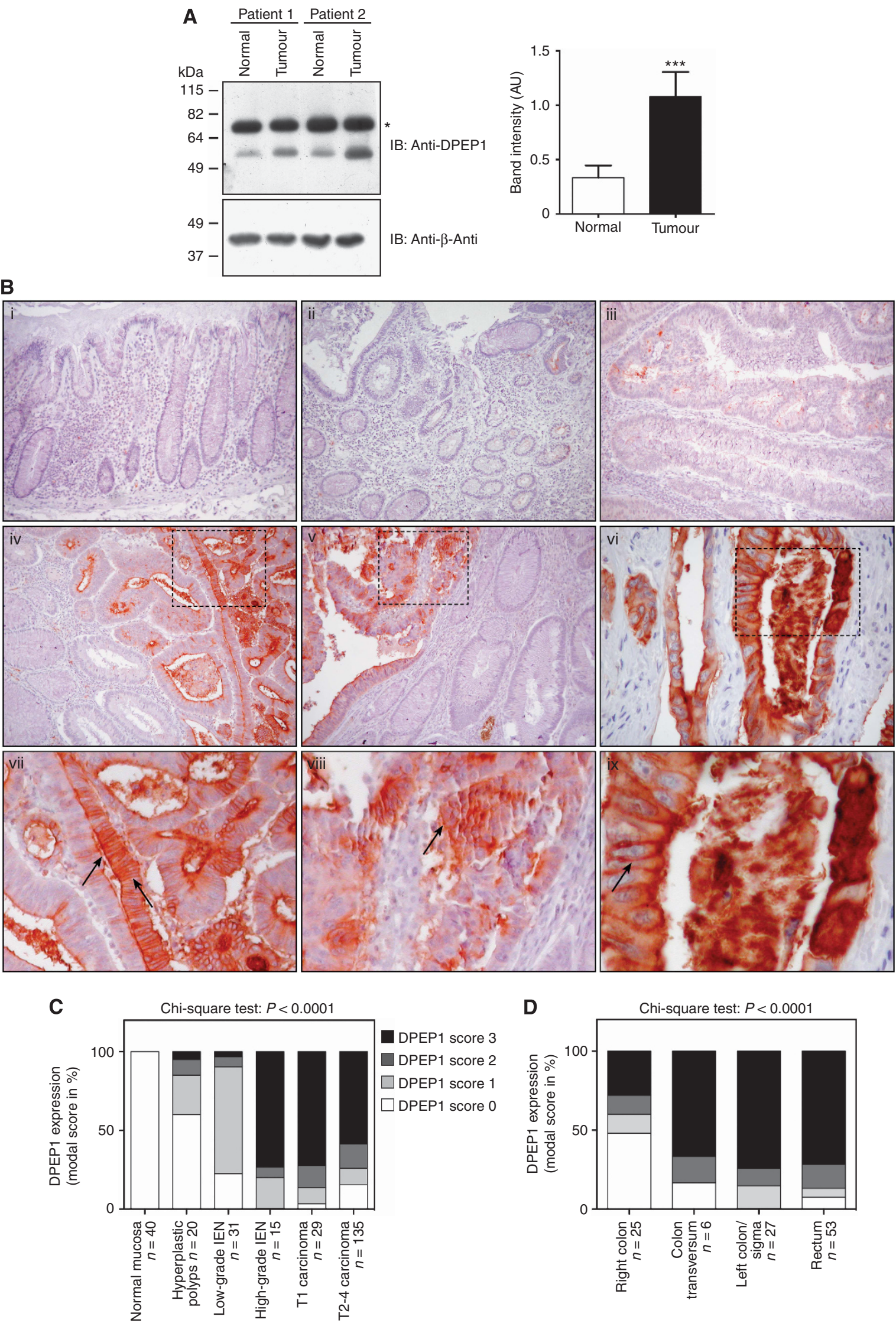
silencing of DPEP1 expression in CRC cell lines exhibited no effect on cell growth or apoptosis, an inhibitory effect on tumour invasion through a matrigel matrix has been observed (Toiyama et al, 2011). Accordingly, we have shown that DPEP1 expression did not correlate with the expression of the proliferation marker Ki67 (Supplementary Figure 4) and we were further not able to detect a ubiquitous upregulation of the lipoxygenase pathway (data not shown). Dipeptidase 1 is a GPI-anchored protein containing a sn-1,2-diacylglycerol (DAG) structure embedded within the plasma membrane. The PLC-dependent cleavage results in the secretion of the protein revealing a cross-reacting determinant (CRD) and a DAG structure remaining within the plasma membrane. Indeed, we observed an increase in shed DPEP1 in tumour samples by detecting increased CRD levels (data not shown), leaving the DAG-like structure of the GPI anchor as a potential second messenger. How exactly DPEP1 expression is linked to CRC progression mechanistically remains to be evaluated

\begin{tabular}{|c|c|c|c|c|}
\hline Variable & TMA & $\begin{array}{c}\text { Stage } \\
\text { analysis }\end{array}$ & $\begin{array}{c}\text { Localisation } \\
\text { analysis }\end{array}$ & $\begin{array}{l}\text { Survival } \\
\text { analysis }\end{array}$ \\
\hline Total cases & 148 & 142 & 111 & 126 \\
\hline Sex (male \%) & 45.27 & 45.77 & 44.14 & 45.24 \\
\hline $\begin{array}{l}\text { Age - mean } \\
\text { years (s.d.) }\end{array}$ & $66.11(11.68)$ & 66.25 (11.43) & $66.67(10.81)$ & $65.63(11.48)$ \\
\hline \multicolumn{5}{|c|}{ Tumour stage $^{a}(\%)$} \\
\hline $\begin{array}{l}\text { T1 } \\
\text { T2 } \\
\text { T3 } \\
\text { T4 }\end{array}$ & $\begin{array}{r}4.73 \\
18.92 \\
65.54 \\
10.14\end{array}$ & $\begin{array}{c}4.93 \\
19.01 \\
66.2 \\
9.9\end{array}$ & $\begin{array}{r}5.41 \\
18.92 \\
66.67 \\
9\end{array}$ & $\begin{array}{r}4.8 \\
18.4 \\
67.2 \\
9.6\end{array}$ \\
\hline \multicolumn{5}{|c|}{ Lymph-node metastasis $^{\mathrm{a}}(\%)$} \\
\hline $\begin{array}{l}\text { N0 } \\
\text { N1/2 }\end{array}$ & $\begin{array}{l}46.62 \\
52.7\end{array}$ & $\begin{array}{l}47.18 \\
52.825\end{array}$ & $\begin{array}{l}45.95 \\
54.05\end{array}$ & $\begin{array}{l}43.2 \\
56.8\end{array}$ \\
\hline \multicolumn{5}{|l|}{ Localisation } \\
\hline $\begin{array}{l}\text { Right colon } \\
\text { Colon } \\
\text { transversum } \\
\text { Left colon/ } \\
\text { Sigma } \\
\text { Rectum }\end{array}$ & $\begin{array}{c}25 \\
6 \\
29 \\
55\end{array}$ & $\begin{array}{c}25 \\
6 \\
27 \\
53\end{array}$ & $\begin{array}{c}25 \\
6 \\
27 \\
53\end{array}$ & $\begin{array}{c}20 \\
6 \\
26 \\
52\end{array}$ \\
\hline $\begin{array}{l}\text { Abbreviation: TI } \\
{ }^{{ }^{a} \text { Data for tumou }} \\
\text { TMA set; sex inf }\end{array}$ & $\begin{array}{l}=\text { tissue microar } \\
\text { tage and lymph } \\
\text { mation missing fo }\end{array}$ & $\begin{array}{l}\text { rray. } \\
\text { node metastasis a } \\
\text { or two patients. }\end{array}$ & missing for one & atient in the total \\
\hline
\end{tabular}

in future studies, for example, in in vivo models. An insight into the mechanistic rational underlying DPEP1 expression may also help to explain tissue-specific differences in DPEP1 regulation, as manifest in an increased DPEP1 expression in CRC (Toiyama et al, 2011; and this study) vs a decreased DPEP1 expression in pancreatic cancer (Zhang et al, 2012).

In particular, patients with chronic inflammations including Crohn's disease and ulcerative colitis (UC) have been associated with an increased incidence of CRC development and are thus required to undergo preventive and continuous colonoscopic surveillance (Bansal and Sonnenberg, 1996; Solomon and Schnitzler, 1998; Eaden et al, 2002; Itzkowitz et al, 2005). In one of the most comprehensive studies linking over 3000 UC cases to CRC progression, the HR for death was 5.7 (95\% CI: 4.6-7.0) (Ekbom et al, 1990). An effective risk assessment and subsequent CRC prevention in those patients relies on the identification of high-grade IEN as a risk hazard

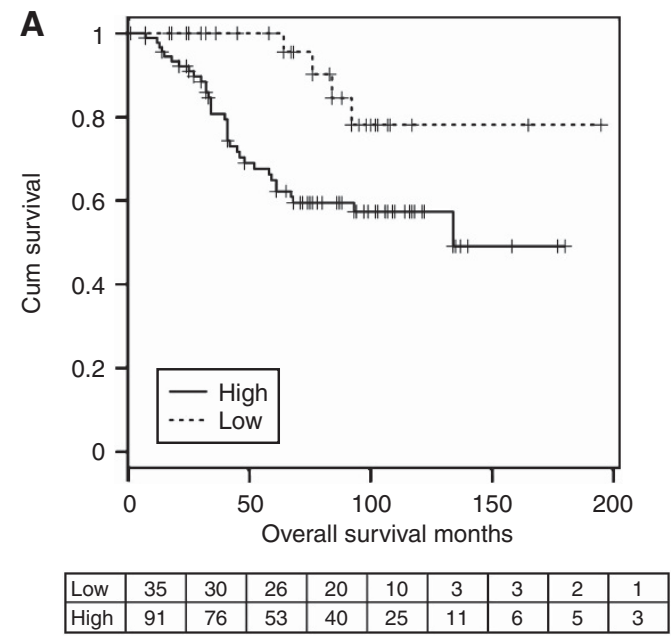

B

\begin{tabular}{l|ccc}
\hline Variable & Hazard ratio (HR) & \multicolumn{1}{c}{$95 \% \mathrm{Cl}$ for (HR) } & $P$-value \\
\hline \hline DPEP1 intensity (low vs high) & 0.3116 & $0.1097-0.8849$ & 0.0285 \\
Tumour stage (T3/4 vs TO-2) & 1.62205 & $0.6742-3.9024$ & 0.2802 \\
Lymp-node metastasis (N1/2 vs NO) & 0.00363 & $0.00019-0.0676$ & 0.0002 \\
\hline
\end{tabular}

Figure 5. DPEP1 protein expression is an independent prognostic marker for CRC patient outcome. (A) Overall survival analysis was performed with $126 \mathrm{CRC}$ cases (validation cohort 2). Variables were evaluated and plotted by constructing Kaplan-Meier survival curves. The plot for DPEP1 staining intensity (score $0 / 1$ vs $2 / 3$ ) is shown with $P=0.0106$ (LR). The number of patients at risk at each given time point is shown below the plot. (B) Variables that were positive in univariable tests were used to construct a multivariable cox regression model. DPEP1 intensity and lymph-node metastasis were independent predictors of CRC patient outcome.

Figure 4. High DPEP1 protein expression correlates with high-grade IEN and tumour localisation. (A) Protein was extracted from tumour and noncancerous tissue of multiple patients. The cell lysates of two representative patient samples were immunoblotted with an anti-DPEP1 and an anti- $\beta$ actin antibody. The semi-quantitative analyses of band intensities, using immunoblots of overall 13 paired patient tissue samples, are shown \pm s.e. with $\left.P<0.0001{ }^{(\star \star}\right)$, Student's t-test. *, non-specific band. AU, arbitrary units; IB, Immunoblot. (B) Immunohistochemical analysis of DPEP1 expression in surgical specimens of $\mathrm{CR}$ epithelia during tumour progression. Arrows indicate the apical and circumferential staining pattern of DPEP1. (i) Normal colonic mucosa, (ii) hyperplastic polyp, (iii) tubular adenoma with low-grade dysplasia, (iv) tubular adenoma with high-grade dysplasia, (v) transition between low-grade to high-grade dysplasia and carcinoma as well as (vi) carcinoma. Panels (vii-ix) show magnified portions of each image as indicated (dashed squares). (C) DPEP1 expression in correlation with tumour stage was analysed using FFPEs of normal mucosa $(n=40)$, hyperplastic polyps $(n=20)$, adenomas with low-grade IEN $(n=31)$, adenomas with high-grade IEN $(n=15)$, T1 carcinomas $(n=22)$ as well as the CRC-TMA bearing T1 $(n=7)$ and T2-T4 carcinomas $(n=135)$. (D) DPEP1 expression in correlation with tumour localisation was analysed using the CRC-TMA with clinical specimen of right colon $(n=25)$, colon transversum $(n=6)$, left colon/Sigma $(n=27)$ and rectum $(n=53)$. Cases with missing information concerning CRC localisation were omitted. 
with subsequent prophylactic colectomy (Itzkowitz et al, 2005; Campbell and Maxwell, 2009). However, endoscopic identification of dysplasia in UC patients remains difficult due to diffuse or multifocal pathology and the lack of macroscopic markers specific for high-grade IEN. In the present study, we have identified DPEP1 as a highly specific marker of high-grade IEN and CRCs. On-going improvements in the field of gastrointestinal endoscopic surveillance including real-time histology, endocytoscopy, chromoscopy and confocal laser endomicroscopy (Kiesslich et al, 2007; Li et al, 2010) may in the future provide a routine diagnostic platform to screen for DPEP1 in vivo as a predictive marker for high-grade IEN. Using DPEP1's enzymatic activity or its shed CRD for visualising highgrade IEN as potential diagnostic determinants in vivo may improve the efficacy of endoscopic surveillance.

In conclusion, we have provided evidence that DPEP1 expression is highly increased in CRC. We report for the first time that DPEP1 is prominently upregulated during transition from low-grade to high-grade IEN, being a promising marker for screening approaches, for example, for patients suffering from UC. Thus, DPEP1 became an attractive candidate for further molecular studies due to its adverse effect on prognosis of CRC patients.

\section{ACKNOWLEDGEMENTS}

This work was partially funded by Merck KGaA (Darmstadt, Germany). We thank Prof. Nigel Hooper (University of Leeds, UK) for donation of the anti-DPEP1 antibody as well as Sonja Vollbehr and Bianca Zinke (Institute for Experimental Cancer Research, UK $\mathrm{S}-\mathrm{H}$ Campus Kiel, Germany) for technical support. We are furthermore grateful to the late Prof. Siegfried Matzku, who initiated this study with outstanding enthusiasm and expertise. In addition, we thank Prof. Bernd Kremer, former head of the Department of General Surgery, for his clinical and scientific support.

\section{CONFLICT OF INTEREST}

The authors declare no conflict of interest.

\section{REFERENCES}

Austruy E, Cohen-Salmon M, Antignac C, Beroud C, Henry I, Nguyen VC, Brugieres L, Junien C, Jeanpierre C (1993) Isolation of kidney complementary DNAs down-expressed in Wilms' tumor by a subtractive hybridization approach. Cancer Res 53(12): 2888-2894.

Bansal P, Sonnenberg A (1996) Risk factors of colorectal cancer in inflammatory bowel disease. Am J Gastroenterol 91(1): 44-48.

Campbell FC, Maxwell RJ (2009) Rationale for cancer prevention strategies in high-risk ulcerative colitis. Surgeon 7(2): 96-100.

Duffy MJ, van Dalen A, Haglund C, Hansson L, Holinski-Feder E, Klapdor R, Lamerz R, Peltomaki P, Sturgeon C, Topolcan O (2007) Tumour markers in colorectal cancer: European Group on Tumour Markers (EGTM) guidelines for clinical use. Eur J Cancer 43(9): 1348-1360.

Eaden JA, Mayberry JF. British Society for Gastroenetrology; Association of Coloproctology for Great Britain, Ireland (2002) Guidelines for screening and surveillance of asymptomatic colorectal cancer in patients with inflammatory bowel disease. Gut 51(Suppl 5): V10-V12.

Eisenach PA, de Sampaio PC, Murphy G, Roghi C (2012) Membrane type 1 matrix metalloproteinase (MT1-MMP) ubiquitination at Lys581 increases cellular invasion through type I collagen. J Biol Chem 287(14): 11533-11545.

Ekbom A, Helmick C, Zack M, Adami HO (1990) Ulcerative colitis and colorectal cancer. A population-based study. N Engl J Med 323(18): 1228-1233.

Fearon ER, Vogelstein B (1990) A genetic model for colorectal tumorigenesis. Cell 61(5): 759-767.
Green AR, Krivinskas S, Young P, Rakha EA, Paish EC, Powe DG, Ellis IO (2009) Loss of expression of chromosome 16q genes DPEP1 and CTCF in lobular carcinoma in situ of the breast. Breast Cancer Res Treat 113(1): 59-66.

Harrison S, Benziger H (2011) The molecular biology of colorectal carcinoma and its implications: a review. Surgeon 9(4): 200-210.

Henderson Jr. WR (1994a) Role of leukotrienes in asthma. Ann Allergy 72(3): $272-278$.

Henderson Jr. WR (1994b) The role of leukotrienes in inflammation. Ann Intern Med 121(9): 684-697.

Itzkowitz SH, Present DH. Crohn's, Colitis Foundation of America Colon Cancer in IBDSG (2005) Consensus conference: colorectal cancer screening and surveillance in inflammatory bowel disease. Inflamm Bowel Dis 11(3): 314-321.

Jemal A, Ward E, Thun M (2010) Declining death rates reflect progress against cancer. PLoS One 5(3): e9584.

Kiesslich R, Goetz M, Lammersdorf K, Schneider C, Burg J, Stolte M, Vieth M, Nafe B, Galle PR, Neurath MF (2007) Chromoscopy-guided endomicroscopy increases the diagnostic yield of intraepithelial neoplasia in ulcerative colitis. Gastroenterology 132(3): 874-882.

Kozak EM, Tate SS (1982) Glutathione-degrading enzymes of microvillus membranes. J Biol Chem 257(11): 6322-6327.

Kropp H, Sundelof JG, Hajdu R, Kahan FM (1982) Metabolism of thienamycin and related carbapenem antibiotics by the renal dipeptidase, dehydropeptidase. Antimicrob Agents Chemother 22(1): 62-70.

Li Z, Yu T, Zuo XL, Gu XM, Zhou CJ, Ji R, Li CQ, Wang P, Zhang TG, Ho KY, Li YQ (2010) Confocal laser endomicroscopy for in vivo diagnosis of gastric intraepithelial neoplasia: a feasibility study. Gastrointest Endosc 72(6): 1146-1153.

McIver CM, Lloyd JM, Hewett PJ, Hardingham JE (2004) Dipeptidase 1: a candidate tumor-specific molecular marker in colorectal carcinoma. Cancer Lett 209(1): 67-74.

Nakagawa H, Inazawa J, Inoue K, Misawa S, Kashima K, Adachi H, Nakazato H, Abe T (1992) Assignment of the human renal dipeptidase gene (DPEP1) to band q24 of chromosome 16. Cytogenet Cell Genet 59(4): $258-260$.

Okamoto T, Matsumura N, Mandai M, Oura T, Yamanishi Y, Horiuchi A, Hamanishi J, Baba T, Koshiyama M, Shiozawa T, Konishi I (2011) Distinguishing primary from secondary mucinous ovarian tumors: an algorithm using the novel marker DPEP1. Mod Pathol 24(2): 267-276.

Schneider CA, Rasband WS, Eliceiri KW (2012) NIH Image to ImageJ: 25 years of image analysis. Nat Methods 9(7): 671-675.

Siegel R, DeSantis C, Virgo K, Stein K, Mariotto A, Smith T, Cooper D, Gansler T, Lerro C, Fedewa S, Lin C, Leach C, Cannady RS, Cho H, Scoppa S, Hachey M, Kirch R, Jemal A, Ward E (2012) Cancer treatment and survivorship statistics, 2012. CA Cancer J Clin 62(4): 220-241.

Siegel R, Ward E, Brawley O, Jemal A (2011) Cancer statistics, 2011: the impact of eliminating socioeconomic and racial disparities on premature cancer deaths. CA Cancer J Clin 61(4): 212-236.

Solomon MJ, Schnitzler M (1998) Cancer and inflammatory bowel disease: bias, epidemiology, surveillance, and treatment. World J Surg 22(4): 352-358.

Strausberg RL (2001) The Cancer Genome Anatomy Project: new resources for reading the molecular signatures of cancer. J Pathol 195(1): 31-40.

R Core Team (2012) R: A language and environment for statistical computing. R Foundation for Statistical Computing: Vienna, Austria, ISBN 3-900051-07-0. Available at http://www.R-project.org/.

Therneau T (2012) A Package for Survival Analysis in S. R package version 2.37-2. Available at http://CRAN.R-project.org/package=survival.

Toiyama Y, Inoue Y, Yasuda H, Saigusa S, Yokoe T, Okugawa Y, Tanaka K, Miki C, Kusunoki M (2011) DPEP1, expressed in the early stages of colon carcinogenesis, affects cancer cell invasiveness. J Gastroenterol 46(2): 153-163.

Wolpin BM, Mayer RJ (2008) Systemic treatment of colorectal cancer. Gastroenterology 134(5): 1296-1310.

Yajima S, Ishii M, Matsushita H, Aoyagi K, Yoshimatsu K, Kaneko H, Yamamoto N, Teramoto T, Yoshida T, Matsumura Y, Sasaki H (2007) Expression profiling of fecal colonocytes for RNA-based screening of colorectal cancer. Int J Oncol 31(5): 1029-1037.

Zhan X, Desiderio DM (2010) Signaling pathway networks mined from human pituitary adenoma proteomics data. BMC Med Genomics 3: 13 . 
Zhang G, Schetter A, He P, Funamizu N, Gaedcke J, Ghadimi BM, Ried T, Hassan R, Yfantis HG, Lee DH, Lacy C, Maitra A, Hanna N, Alexander HR, Hussain SP (2012) DPEP1 inhibits tumor cell invasiveness, enhances chemosensitivity and predicts clinical outcome in pancreatic ductal adenocarcinoma. PLoS One 7(2): e31507.
This work is published under the standard license to publish agreement. After 12 months the work will become freely available and the license terms will switch to a Creative Commons AttributionNonCommercial-Share Alike 3.0 Unported License.

Supplementary Information accompanies this paper on British Journal of Cancer website (http://www.nature.com/bjc) 